\title{
NEW APPROXIMATE SOLUTIONS PER UNIT OF TIME FOR PERIODICALLY CHECKED SYSTEMS WITH DIFFERENT LIFETIME DISTRIBUTIONS
}

\author{
J. RODRIGUES DIAS
}

Received 26 October 2005; Revised 19 September 2006; Accepted 24 September 2006

Systems with different lifetime distributions, associated with increasing, decreasing, constant, and bathtub-shaped hazard rates, are examined in this paper. It is assumed that a failure is only detected if systems are inspected. New approximate solutions for the inspection period and for the expected duration of hidden faults are presented, on the basis of the assumption that only periodic and perfect inspections are carried out. By minimizing total expected cost per unit of time, on the basis of numerical results and a range of comparisons, the conclusion is drawn that these new approximate solutions are extremely useful and simple to put into practice.

Copyright (C) 2006 J. Rodrigues Dias. This is an open access article distributed under the Creative Commons Attribution License, which permits unrestricted use, distribution, and reproduction in any medium, provided the original work is properly cited.

\section{Introduction}

Let us consider a system, which may be of any type or nature, whose lifetime is a continuous random variable $T$ with density function $f(t)$ and expected value $E(T)$. If $F(t)$ is the respective distribution function, then the reliability function $R(t)$ and the hazard rate $h(t)$ are given by

$$
R(t)=1-F(t) ; \quad h(t)=\frac{f(t)}{R(t)} .
$$

Let us assume that a perfect or imperfect system state is only detected if the system is inspected and that inspections are perfect and periodic.

We will regard a cycle as the interval of time between the initial instant and the instant at which a failure is detected and corrected by means of an inspection. Let us assume that system state is not changed by inspections and that their duration is nil. A failure having been detected and corrected, we will assume that the system is as good as new; a renewal 
process thus takes place and a new cycle begins. In this paper, we will analyze the system operation per unit of time over the duration of a cycle.

The proposed solutions that will be presented in this paper are applicable in a wide variety of fields. Engineering, medicine, and maintenance scenarios would benefit from these procedures where the goal is the minimization of expected costs in systems whose lifetime is a random variable and where failures may occur. For example, they could be applied in the detection of

(a) failures in nuclear systems;

(b) enemy missiles or airplanes;

(c) forest fires and fires in buildings;

(d) global or species-specific diseases affecting people, birds, and other animals; current developments and recent experience highlight the importance of finding such solutions.

If $N$ is the number of system inspections per cycle (including the inspection at whose instant a failure is detected), and if $D$ represents the detection time of a hidden fault (between the instant of system failure and the instant of its detection during the next inspection), which are both random variables, while $P$ is the inspection period (with inspection times over $P, 2 P, 3 P, \ldots)$, then their expected values $E(\cdot)$ are given by

$$
E(N)=\sum_{k=0}^{\infty}(k+1) \int_{k P}^{(k+1) P} f(t) d t=\sum_{k=0}^{\infty} R(k P), \quad E(D)=P \cdot E(N)-E(T) .
$$

If $C_{1}$ is the cost of each inspection and $C_{2}$ is the cost per unit of time of imperfect operation before system failure is detected, then the total expected cost per cycle $E(C)=$ $C_{1} E(N)+C_{2} E(D)$ is minimized for a value of $P$, which is a solution of the following equation (see Rodrigues Dias [7]):

$$
\sum_{k=0}^{\infty} R(k P)-(r+P) \sum_{k=0}^{\infty} k f(k P)=0, \quad r=\frac{C_{1}}{C_{2}}
$$

It should be noted that there may be one, two, or more solutions, which do not depend on the individual values of costs $C_{1}$ and $C_{2}$ (interestingly, they depend on their quotient $r$ ).

This problem was initially presented and examined by Barlow et al. [3].

However, it has been shown that it is extremely difficult or perhaps impossible to analytically obtain a solution for (1.3), even when $T$ has an exponential distribution. Therefore, numerical approaches are required.

In (1.2), a simple and useful geometric interpretation of $E(D)$ is presented. In fact, $E(T)=\int_{0}^{\infty} R(t) d t$ is the area below the reliability function curve $R(t)$ and above the $x$ axis for values between 0 and $\propto$; meanwhile, $P \cdot E(N)=\sum_{k=0}^{+\infty} P \cdot R(k P)$ is the sum of the area of the rectangles whose base is $P$ centered on $k P(k=0,1,2, \ldots)$ and whose height is $R(k P)$. Therefore, $E(D)$ is the difference between the latter area and the former area, with regard to $E(T)$. The straightforward conclusion is that $E(D)$ is approximately equal to $P / 2$; however, it may be either larger or smaller than half the inspection period $P$. This 
depends on the shape of the reliability function $R(t)$. There is no general expression which approximates to $E(D)$.

Thus, starting with the simplest approach $E(D) \cong P / 2$ (see Schneeweiss [15]), Nakagawa and Yasui [6] obtained the following approximate solution for the inspection period $P_{C 1}$ per cycle (with $\left.E(N) \cong E(T) / P+1 / 2\right)$ :

$$
P_{C 1}=\sqrt{2 r E(T)}
$$

In a later work (see Rodrigues Dias [8]), in which $T$ has an exponential distribution, based on the analysis of results previously obtained (see Rodrigues Dias [7]), a new approximate solution for the inspection period was obtained by using the least-square method which minimizes the total expected cost per cycle:

$$
P_{C 2}=\frac{\sqrt{2 r E(T)}}{1+0.234 \sqrt{r^{\prime}}}=\frac{P_{C 1}}{G}, \quad G=1+0.234 \sqrt{r^{\prime}} ; r^{\prime}=\frac{r}{E(T)} .
$$

This solution is demonstrated to be nearly optimal because its relative error is approximately equal to zero in terms of costs. It can be regarded as a generalization of the approach developed by Nakagawa and Yasui [6], where $G$ is the generalization factor.

The practical usefulness of simple approaches should be stressed, as can be seen in a quality control context of Saniga and Shirland in [14].

Problems of the same type can be found in Badía et al. [2], Dieulle [4], Rodrigues Dias [9], Rodrigues Dias and Infante [12], Sheu et al. [16], and Vaurio [17], for example. In Rodrigues Dias $[10,11]$, two new approaches dealing with different sampling intervals in quality control are proposed.

\section{A new approximate solution for the inspection period}

Let us now discuss whether it is possible to obtain solutions which in some way correspond to those previously presented in (1.4) and (1.5), with a view to minimizing expected total cost per unit of time of the cycle. In fact, the duration of each cycle is a random variable, resulting from system lifetime $T$ plus the duration of a hidden fault $D$.

Hence, if $C_{U}$ is the total cost per unit of time and $E\left(C_{U}\right)$ is its expected value, and there is a renewal process (see Ross [13]), we have

$$
E\left(C_{U}\right)=\frac{C_{1} E(N)+C_{2} E(D)}{E(T)+E(D)} .
$$

What must be determined is the value of period $P$ that minimizes the function given by (2.1). With regard to the minimization of expected total cost per cycle, in general, an analytical solution cannot be obtained. Therefore, it is essential that approximate solutions be obtained and that an evaluation be carried out, for different situations, as to whether they are satisfactory from a practical point of view. 
Let us suppose that $E(D) \cong P / 2$. If $E(N) \cong E(T) / P+1 / 2$, the following expression can easily be obtained:

$$
E\left(C_{U}\right) \cong \frac{2 C_{1} E(T)+C_{1} P+C_{2} P^{2}}{2 P E(T)+P^{2}} .
$$

By calculating the respective derivative associated with $P$ and making it equal to zero, the following second-order equation can be obtained:

$$
\left[2 C_{2} E(T)-C_{1}\right] \cdot P^{2}-4 C_{1} E(T) \cdot P-4 C_{1} E(T)^{2}=0 .
$$

As one of the two solutions will always be negative, the other one can be expressed as follows:

$$
P=\frac{[2 r E(T)+2 E(T) \cdot \sqrt{2 r E(T)}]}{[2 E(T)-r]} .
$$

By means of a suitable convenient algebraic transformation, assuming for instance an auxiliary variable $z$, such that $z^{2}=2 r E(T)$, the simple solution $P_{U 1}$ can be obtained:

$$
P_{U 1}=\frac{2 r E(T)}{\sqrt{2 r E(T)}-r}=\frac{P_{C 1}^{2}}{P_{C 1}-r} .
$$

Thus, $P_{U 1}$ represents the first approximate solution presented in this paper for period $P$ that minimizes expected total cost per unit of time.

Interestingly, it is possible to express $P_{U 1}$ as a function of $P_{C 1}$ and $r$. Even more interestingly, when $r$ tends towards zero (which may mean increasingly lower costs for each inspection), the two expressions $P_{U 1}$ and $P_{C 1}$ get closer together. In a sense, it can be said that the approximate solution we have obtained, given by $(2.5)$, is a generalization of the approach given in (1.4).

As a simple example, let us consider a system with an average lifetime $E(T)=1000$ hours, the numeric value of each inspection cost $C_{1}$ being equal to 1 and the numeric value of cost per hour of imperfect operation $C_{2}$ being equal to 20. Thus, very simply, we have $r=0.05$ and from (2.5), we can calculate that $P_{U 1} \cong 10.05$ hours. It should be noted that no assumptions are made here about the type of distribution of $T$.

This new approximate solution will be analyzed in the following section.

\section{Results obtained and analysis of results}

We will present some numerical results and analyze, in brief, whether the new approximate solution for the inspection period $P_{U 1}$ can be regarded as satisfactory. To this end, we will consider different situations. Firstly, let us attribute a different degree of significance to the cost of each inspection $C_{1}$, comparing each with the cost per unit of time of imperfect operation $C_{2}$. In fact, we will use different values of $r^{\prime}$, given in (1.5), which is a dimensionless parameter, as can be seen in the following tables. This means taking $E(T)$ as the unit of time. Secondly, we will consider the case in which the system has different failure rates $h(t)$, for three different lifetime distributions: (a) the Weibull, (b) the log-normal, and (c) the Hjorth [5] distributions. 
(a) The Weibull distribution is a model very often used in this kind of reliability context. Interestingly, this distribution, whose shape parameter is equal to 2.5 , is used to model the incubation period of the HIV1virus (see Amaral et al. [1]).

In the Weibull distribution, $\alpha$ being a scale parameter and $\beta$ a form parameter, the reliability function is given by

$$
R(t)=\exp \left(\frac{-t}{\alpha}\right)^{\beta}, \quad t \geq 0 .
$$

In this paper, let us assume that form parameter $\beta$ has the following values: $0.7,1,2,3,4$, and 5. System hazard rate $h(t)$ is decreasing where it is less than 1 , and where it is greater than $1, h(t)$ is increasing. When $\beta=1$, we have an exponential distribution corresponding to a constant hazard rate $h(t)=1 / \alpha$. Finally, when the form parameter is close to 3 , there is a degree of approach to normal distribution.

(b) In a system in which the lifetime distribution is log-normal, we have

$$
R(t)=1-\Phi\left(\frac{\ln (t)-\mu}{\sigma}\right), \quad t \geq 0
$$

where $\Phi(\cdot)$ is the standard normal distribution function and $\mu$ and $\sigma$ are two parameters. Interestingly, with the log-normal distribution, $h(t)$ increases initially but then decreases.

(c) The Hjorth [5] lifetime distribution, depending on 3 parameters $\delta, \gamma$, and $\theta$, has a reliability function given by

$$
R(t)=\exp \left(-\frac{\delta t^{2}}{2}\right) \cdot(1+\gamma t)^{-\theta / \gamma}, \quad t \geq 0
$$

This is significant because $h(t)$ can present a range of very different shapes. In particular, if $\theta=\gamma=1$, and if $\delta=0.01$, then $h(t)$ is bathtub-shaped.

Thus, the above three distributions allow us to consider all possible shapes of system failure rate $h(t)$.

Now, let us begin by comparing the approach $P_{U 1}$ given by $(2.5)$ with the exact solution $P_{U}$ obtained minimizing $E\left(C_{U}\right)$ given by $(2.1), E(N)$ and $E(D)$ being obtained from (1.2). Usually, when $E(N)$ increases, $E(D)$ decreases. This "exact" solution is calculated using numerical methods. Thus, we consider the quotient $Q_{1}$, given by the following expression, which gives the deviation, in relative terms and in percentage terms, between the two solutions for the inspection period:

$$
Q_{1}=\frac{P_{U 1}-P_{U}}{P_{U}} \cdot 100 \%
$$

Secondly, let us analyze the sensitivity of $E\left(C_{U}\right)$ as a function of $P$, in terms of evaluating the validity of the approximate solution $P_{U 1}$ in practical terms. To this end, let us consider $Q_{2}$, which gives the relative error, as a percentage, of the expected total cost associated with the solution $P_{U 1}$, when compared with minimum cost, for the exact 
Table 3.1. Values of relative errors $Q_{1}, Q_{2}$, and $Q_{3}$, in percentages, for different values of $\beta$ of the Weibull distribution and for four values of $r^{\prime}$.

\begin{tabular}{l|rrr|rrr|rrr|rrr}
\hline & \multicolumn{3}{|c|}{$r^{\prime}=0.0125$} & \multicolumn{3}{c|}{$r^{\prime}=0.0250$} & \multicolumn{3}{c|}{$r^{\prime}=0.0500$} & \multicolumn{3}{c}{$r^{\prime}=0.1000$} \\
\hline$\beta$ & $Q_{1}$ & $Q_{2}$ & $Q_{3}$ & $Q_{1}$ & $Q_{2}$ & $Q_{3}$ & $Q_{1}$ & $Q_{2}$ & $Q_{3}$ & $Q_{1}$ & $Q_{2}$ & $Q_{3}$ \\
\hline 0.7 & 7.3 & 0.2 & 8.4 & 9.0 & 0.3 & 10.5 & 11.2 & 0.5 & 13.1 & 13.6 & 0.6 & 16.4 \\
1 & 2.7 & 0.0 & 2.7 & 3.9 & 0.1 & 3.9 & 5.7 & 0.1 & 5.6 & 8.3 & 0.2 & 8.1 \\
2 & 0.0 & 0.0 & 0.0 & 0.0 & 0.0 & 0.0 & 0.0 & 0.0 & 0.0 & 0.0 & 0.0 & 0.0 \\
3 & 0.0 & 0.0 & 0.0 & 0.0 & 0.0 & 0.0 & -0.1 & 0.0 & -0.1 & -0.6 & 0.0 & -0.2 \\
4 & 0.0 & 0.0 & 0.0 & 0.0 & 0.0 & 0.0 & 0.1 & 0.0 & 0.0 & -7.2 & 0.2 & -1.0 \\
5 & 0.0 & 0.0 & 0.0 & 0.0 & 0.0 & 0.0 & -3.2 & 0.0 & 0.0 & -8.0 & 1.1 & -5.0 \\
\hline
\end{tabular}

solution $P_{U}$ :

$$
Q_{2}=\frac{E\left(C_{U 1}\right)-E\left(C_{U}\right)}{E\left(C_{U}\right)} \cdot 100 \%
$$

Thirdly, as $P_{U 1}$ was obtained by considering $E(D) \cong P / 2$, we are keen to evaluate this assumption. Thus, with $E\left(D_{U}\right)$ as the expected detection time of a system failure and $P_{U}$ the optimum inspection period, we define $Q_{3}$ as

$$
Q_{3}=\frac{E\left(D_{U}\right)-P_{U} / 2}{E\left(D_{U}\right)} \cdot 100 \%
$$

Results obtained for $Q_{1}, Q_{2}$, and $Q_{3}$ are shown in Table 3.1, for the Weibull distribution, for four values of $r^{\prime}$.

It can be shown that for values of $\beta$ which approximate to 2,3 , and 4 , if $r^{\prime}$ is not great (which means a very low expected number of inspections per cycle, which is unusual in practical applications), the relative errors of $P_{U 1}$ are practically nil in absolute values: they do not exceed $0.1 \%\left(r^{\prime} \leq 0.05\right)$. The same can be shown for greater values of $\beta$, since $r^{\prime}$ values are not great. Meanwhile, when the system failure rate is constant $(\beta=1)$, it can be shown that values of $Q_{1}$ are significant, increasing whenever $r^{\prime}$ increases.

Now, let us analyze what happens with $Q_{2}$. It can be easily shown that the relative errors of expected total costs per unit of time are almost always nil (to one decimal place). Interestingly, even when the value of $Q_{1}$ is $13.6 \%$, the corresponding value of $Q_{2}$ does not exceed $0.6 \%$. This means that $E\left(C_{U}\right)$ shows a low degree of sensitivity to variations in period $P$, which clearly increases the significance of approximate solutions, especially as they are simple and can be rapidly obtained.

To conclude our analysis of the $P_{U 1}$ approach, let us see what happens with $Q_{3}$. It can typically be shown that the better the approximation $E(D) \approx P / 2$ is, the better the approach given by $(2.5)$ is. At the same time, it can be shown that $E(D)$ may be larger or smaller than $P / 2$, depending on the distribution used. Meanwhile, in the case of exponential distributions, it is always greater, increasing as the value of $r^{\prime}$ increases.

Now, in Table 3.2, with regard to the log-normal and Hjorth distributions, relative errors $Q_{1}, Q_{2}$, and $Q_{3}$ are shown only for 2 values of $r^{\prime}$, since these are sufficient for arriving at a conclusion. 
Table 3.2. Values of the relative errors $Q_{1}, Q_{2}$, and $Q_{3}$, in percentages, for the log-normal and the Hjorth distributions and for $r^{\prime}=0.0125$ and $r^{\prime}=0.0500$.

\begin{tabular}{l|ccr|rrr}
\hline & \multicolumn{3}{|c|}{$r^{\prime}=0.0125$} & \multicolumn{3}{c}{$r^{\prime}=0.0500$} \\
\hline Distribution & $Q_{1}$ & $Q_{2}$ & $Q_{3}$ & $Q_{1}$ & $Q_{2}$ & $Q_{3}$ \\
\hline Log-normal & -0.3 & 0.0 & -0.3 & 2.4 & 0.0 & 0.4 \\
Hjorth & -2.2 & 0.2 & 6.7 & -6.1 & 0.5 & 11.8 \\
\hline
\end{tabular}

Among other possible conclusions, we would point to the following.

(a) Depending on values of $r^{\prime}$ and the system lifetime distribution, values of the $P_{U 1}$ solution may be smaller or larger than those of the exact solution $P_{U}$; the same conclusion may be drawn with regard to $E\left(D_{U}\right)$, if it is compared with $P_{U} / 2$.

(b) The type of lifetime distribution may be of great significance, as can be seen for $r^{\prime}=0.05$.

(c) Finally, values of $Q_{2}$ are small, and may be extremely small; this generally means that approximate solutions for the inspection period are always significant for all shapes of the system hazard rate $h(t)$.

\section{Systems with a constant hazard rate: some new approximate solutions}

In a similar manner to what was done above using (1.5), when the system lifetime is an exponential distribution with a constant hazard rate $h(t)=1 / \alpha$, let us introduce a second new approach $P_{U 2}$ given by

$$
P_{U 2}=\frac{P_{U 1}}{1+0.234 \sqrt{r^{\prime}}}=\frac{P_{U 1}}{G} .
$$

It should be possible to search for another generalization factor which is slightly different from $G$. However, it seems best to keep this one as it has the advantage of being the same as in the case of the solution given by (1.5).

In order to determine its degree of accuracy, let us calculate relative errors $Q_{4}$ :

$$
Q_{4}=\frac{P_{U 2}-P_{U}}{P_{U}} \cdot 100 \%
$$

Some values for $Q_{4}$ are presented in Table 4.1.

As can be seen from the table, with $Q_{4}$ approximately equal to zero, $P_{U 2}$ is a nearly optimal solution for $P_{U}$.

Having concluded that both $P_{C 2}$ and $P_{U 2}$ are nearly optimal solutions when the system has a constant hazard rate, we will now move on to consider the following questions. Are values of $P_{C 2}$ and $P_{U 2}$ very different? In other words, is there a difference in terms of minimizing total expected cost of the cycle and unit of time? If there is a difference, what is its magnitude? In order to provide a quick answer to these questions, let us calculate 
Table 4.1. Values of relative errors $Q_{4}, Q_{5}, Q_{6}, Q_{7}$, and $Q_{8}$, in percentages, for different values of $r^{\prime}$ and $\beta=1$.

\begin{tabular}{l|cccc}
\hline & $r^{\prime}=0.0125$ & $r^{\prime}=0.0250$ & $r^{\prime}=0.0500$ & $r^{\prime}=0.1000$ \\
\hline$Q_{4}$ & 0.1 & -0.2 & -0.4 & -0.8 \\
$Q_{5}$ & 8.6 & 12.6 & 18.8 & 28.8 \\
$Q_{6}$ & -0.0861 & -0.2078 & -0.3879 & -0.7562 \\
$Q_{7}$ & -0.0045 & -0.0152 & 0.0547 & 0.0757 \\
$Q_{8}$ & -0.0104 & -0.0271 & 0.0309 & 0.0278 \\
\hline
\end{tabular}

the relative differences between $P_{U 2}$ and $P_{C 2}$, using $Q_{5}$ given by

$$
Q_{5}=\frac{P_{U 2}-P_{C 2}}{P_{C 2}} \cdot 100 \%=\frac{1}{\sqrt{2 / r^{\prime}}-1} \cdot 100 \% .
$$

Some calculated values are presented in Table 4.1 .

In this case, as in other situations, it is obvious that $Q_{5}$ values show a considerable degree of difference, as do values for optimal periods $P_{C}$ and $P_{U}$, with $P_{U}>P_{C}$. Thus, a key conclusion can now be drawn: it is essential to specify how optimal inspection times are calculated.

Finally, let us look at values of $Q_{1}$ and $Q_{3}$ shown in Table 3.1 when $\beta=1$. It can easily be shown, especially if $r^{\prime}$ is small, that $Q_{1} \cong Q_{3}$. Hence, on the basis of (3.4) and (3.6), we have

$$
\frac{P_{U 1}-P_{U}}{P_{U}} \cong \frac{E\left(D_{U}\right)-P_{U} / 2}{E\left(D_{U}\right)}
$$

As values of $Q_{4}$ are approximately equal to zero, we can consider $P_{U} \cong P_{U 2}$ and on the basis of (4.1), $P_{U 1} \cong G \cdot P_{U}$. Using (4.4), and carrying out some analysis, the approximate solutions $E\left(D_{U}\right)_{1}$ and $E\left(D_{U}\right)_{2}$ can be obtained for the expected duration of hidden faults $E\left(D_{U}\right)$ :

$$
\begin{aligned}
& E\left(D_{U}\right)_{1}=\frac{P_{U}}{2\left(1-0.234 \sqrt{r^{\prime}}\right)}, \\
& E\left(D_{U}\right)_{2}=\frac{P_{U 1}}{2 G\left(1-0.234 \sqrt{r^{\prime}}\right)} .
\end{aligned}
$$

Similarly, considering that in (4.6), we have

$$
G \cdot\left(1-0.234 r^{\prime 1 / 2}\right)=\left(1+0.234 r^{\prime 1 / 2}\right) \cdot\left(1-0.234 r^{\prime 1 / 2}\right) \cong\left(1-0.05 r^{\prime}\right)
$$


then a third simple approximation for $E\left(D_{U}\right)_{3}$ can be derived:

$$
E\left(D_{U}\right)_{3}=\frac{P_{U 1}}{2\left(1-0.05 r^{\prime}\right)}
$$

In order to evaluate how satisfactory these solutions are, let us consider $Q_{6}, Q_{7}$, and $Q_{8}$ given by

$$
\begin{aligned}
Q_{6} & =\frac{E\left(D_{U}\right)_{1}-E\left(D_{U}\right)}{E\left(D_{U}\right)} \cdot 100 \%, \\
Q_{7} & =\frac{E\left(D_{U}\right)_{2}-E\left(D_{U}\right)}{E\left(D_{U}\right)} \cdot 100 \%, \\
Q_{8} & =\frac{E\left(D_{U}\right)_{3}-E\left(D_{U}\right)}{E\left(D_{U}\right)} \cdot 100 \% .
\end{aligned}
$$

Some values are shown in Table 4.1.

From Table 3.1, it can be concluded that $E\left(D_{U}\right)>P_{U} / 2$. Other conclusions can easily be drawn. In all cases, approximate solutions $E\left(D_{U}\right)_{1}, E\left(D_{U}\right)_{2}$, and $E\left(D_{U}\right)_{3}$ can be considered as being nearly optimal. $E\left(D_{U}\right)_{2}$ and $E\left(D_{U}\right)_{3}$ are quite similar approaches in terms of relative error: one of them is better for smaller values of $r^{\prime}$ and the other is better for larger values of $r^{\prime}$. Finally, $E\left(D_{U}\right)_{2}$ contains the constant $G$, which is a possible advantage, and is consistent with other results presented here. Meanwhile, $E\left(D_{U}\right)_{3}$ is certainly the simplest approach.

\section{Conclusions}

In order to minimize the expected total cost of the operation of a system whose operational state can only be determined by means of inspections, it must be inspected either periodically or not. In this paper we consider the case of periodic inspections with two costs: one pertaining to the cost of each inspection and the other pertaining to the cost per unit of time of imperfect operation, following a system failure, before failure is detected and corrected. System lifetime is regarded as a random variable, and several situations with different types of hazard rates are examined, using the Weibull, log-normal, and Hjorth [5] distributions.

In this paper, firstly we review some previous works regarding the minimization of expected total cost per cycle: two approximate solutions for the inspection period are examined.

Secondly, the main point of this paper is presented; by minimizing expected total cost per unit of time, we obtain a new approximate solution for the inspection period. Our results are carefully analyzed for a range of different situations. System lifetime distribution is significant, as it can be seen by means of examples. As our main conclusion, the solution proposed is nearly optimal, for an extremely wide range of contexts, especially if it is considered in terms of costs. At the same time, it is easy to calculate, which is an important point when considering practical applications. In addition, this approximate solution can be regarded as a generalization of a previous solution. These two approaches approximate to each other when the relationship between costs tends towards zero. 
Thirdly, when system hazard rate is constant, we propose a second solution for the inspection period, which is also extremely satisfactory. Using an exponential distribution, three approximate solutions for the expected duration of hidden faults are presented and analyzed using numerical results.

The optimum value of the inspection period may be very different depending on whether the minimization of total expected costs is carried out with respect to a cycle or to the unit of time, especially where the relative significance of inspection costs increases. However, in the opposite situation, the corresponding results are increasingly similar where the expected number of system inspections increases.

Generally, for practical purposes, it is our opinion that the new solutions put forward in this paper can be regarded as extremely satisfactory, and they are also very simple and easy to obtain. In particular, the relative errors of associated total expected costs are almost zero.

\section{Acknowledgments}

The author would like to thank the three referees and the editor in charge for their generous efforts in helping to improve this paper.

\section{References}

[1] J. A. Amaral, M. B. Rosário, and M. T. Paixão, Data and projections of HIV and AIDS in Portugal, Journal of Applied Statistics 27 (2000), no. 3, 269-279.

[2] F. G. Badía, M. D. Berrade, and C. A. Campos, Optimization of inspection intervals based on cost, Journal of Applied Probability 38 (2001), no. 4, 872-881.

[3] R. E. Barlow, L. C. Hunter, and F. Proschan, Optimum checking procedures, Journal of Society for Industrial and Applied Mathematics 11 (1963), no. 4, 1078-1095.

[4] L. Dieulle, Reliability of a system with Poisson inspection times, Journal of Applied Probability 36 (1999), no. 4, 1140-1154.

[5] U. Hjorth, A reliability distribution with increasing, decreasing, constant and bathtub-shaped failure rates, Technometrics 22 (1980), no. 1, 99-107.

[6] T. Nakagawa and K. Yasui, Approximate calculation of inspection policy with Weibull failure times, IEEE Transactions on Reliability 28 (1979), no. 5, 403-404.

[7] J. Rodrigues Dias, Influence de la période d'inspection sur les Couts dans l'inspection périodique des systêmes, Revue de Statistique Appliquée 31 (1983), no. 4, 5-15.

[8] _ A new approximation for the inspection period of systems with different failure rates, European Journal of Operational Research 45 (1990), no. 2-3, 219-223.

[9] - Some approximate inspection policies for a system with imperfect inspections, RAIRO Recherche Opérationnelle 24 (1990), no. 2, 191-199.

[10] _ Analysis of a new method for obtaining different sampling intervals in statistical quality control, Actas do IV Congreso Galego de Estatística e Investigación de Operacións, University of Santiago da Compostela, Spain, 1999, pp. 155-158.

[11] Sampling in quality control with different predetermined intervals: a new approach, Jornadas de Classificação e Análise de Dados (JOCLAD '02), Lisbon, 2002.

[12] J. Rodrigues Dias and P. Infante, Parallel systems with different hazard rates: analysis of inspection periods and costs, Pesquisa Operacional 24 (2004), no. 3, 343-354.

[13] S. M. Ross, Stochastic Processes, 2nd ed., Wiley Series in Probability and Statistics: Probability and Statistics, John Wiley \& Sons, New York, 1996. 
[14] E. M. Saniga and L. E. Shirland, Quality control in practice—a survey, Quality Progress 10 (1977), no. $5,30-33$.

[15] W. G. Schneeweiss, On the mean duration of hidden faults in periodically checked systems, IEEE Transactions on Reliability 25 (1976), no. 5, 346-348.

[16] S. H. Sheu, Y. C. Chen, W. Y. Wang, and N. H. Shin, Economic optimization of off-line inspection with inspection errors, Journal of the Operational Research Society 54 (2003), no. 8, 888-895.

[17] J. K. Vaurio, Optimization of test and maintenance intervals based on risk and cost, Reliability Engineering \& System Safety 49 (1995), no. 1, 23-36.

J. Rodrigues Dias: Department of Mathematics, University of Évora, 7000 Évora, Portugal E-mail address: jrd@uevora.pt 


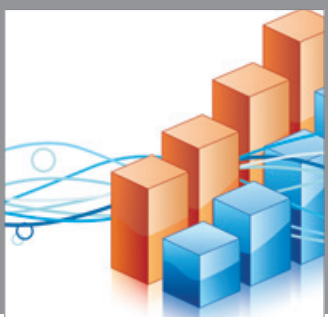

Advances in

Operations Research

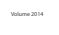

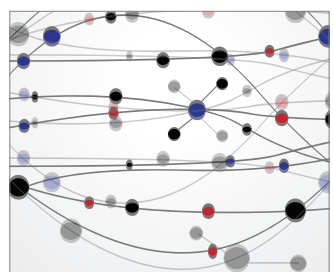

\section{The Scientific} World Journal
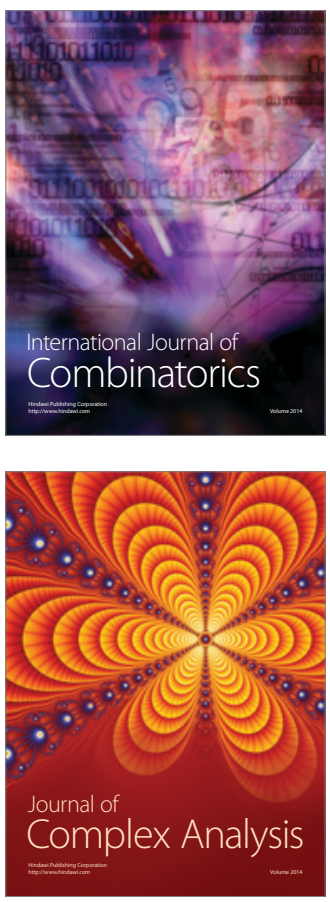

International Journal of

Mathematics and

Mathematical

Sciences
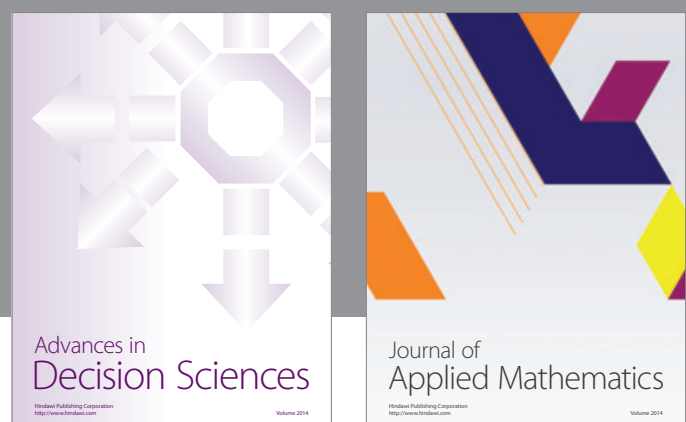

Journal of

Applied Mathematics
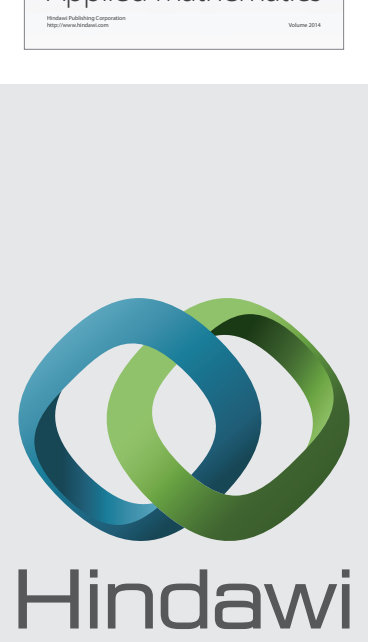

Submit your manuscripts at http://www.hindawi.com
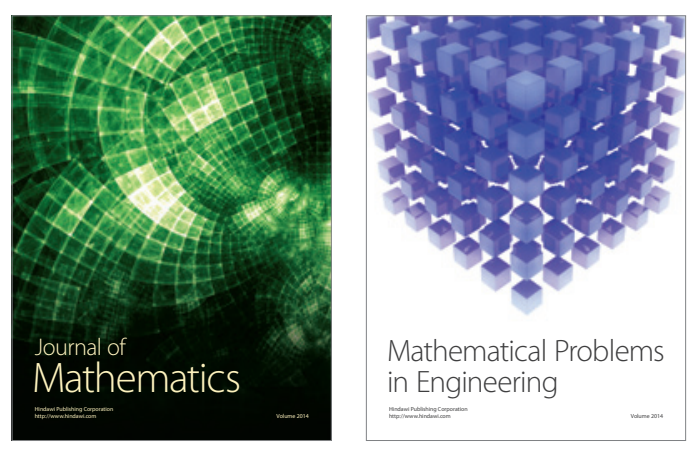

Mathematical Problems in Engineering
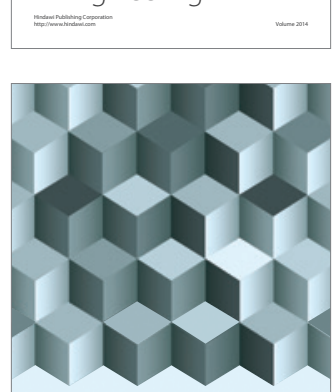

Journal of

Function Spaces
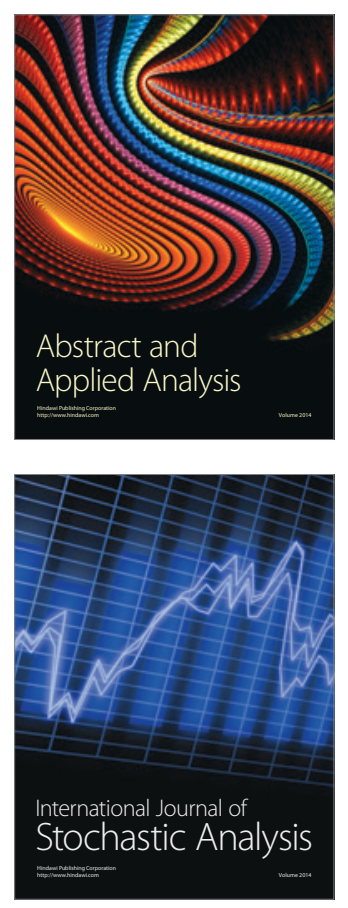

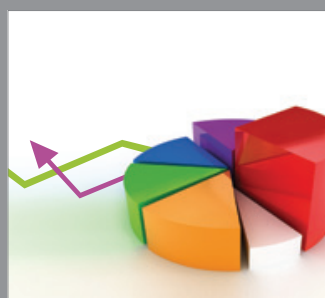

ournal of

Probability and Statistics

Promensencen
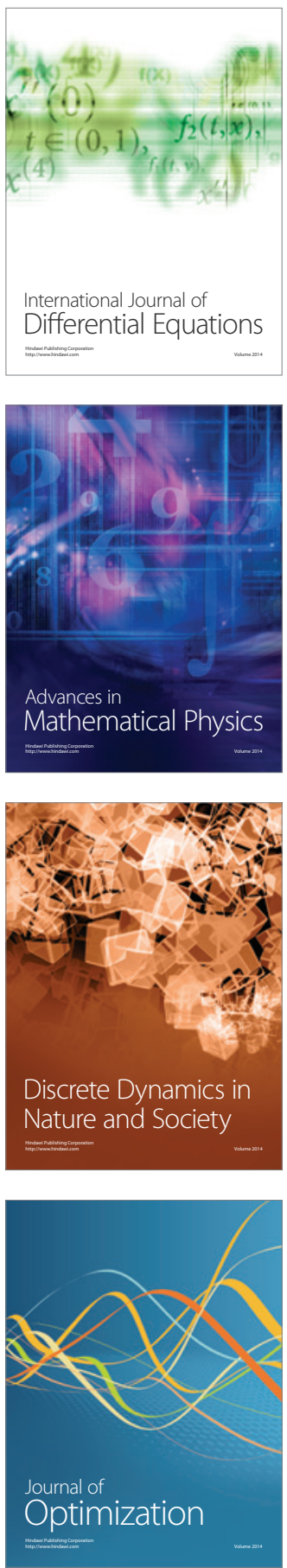